\title{
MicroRNA-146a protects against myocardial ischaemia reperfusion injury by targeting Med1
}

Tiantian Zhang ${ }^{1+}$, Yiwen $\mathrm{Ma}^{2+}$, Lin Gao ${ }^{1}$, Chengyu Mao ${ }^{1}$, Huasu Zeng ${ }^{1}$, Xiaofei Wang ${ }^{1}$, Yapin Sun ${ }^{1}$, Jianmin Gu${ }^{3}$, Yue Wang ${ }^{1}$, Kan Chen ${ }^{1}$, Zhihua Han', Yuqi Fan'1, Jun Gu', Junfeng Zhang ${ }^{1 *}$ and Changqian Wang ${ }^{1 *}$

\author{
*Correspondence: jfzhang_dr@163. \\ com; wcqian@hotmail.com \\ ${ }^{\dagger}$ Tiantian Zhang and Yiwen Ma \\ contributed equally to this work. \\ 'Department of Cardiology, \\ Shanghai Ninth People's Hospital, \\ Shanghai Jiao Tong University \\ School of Medicine, Shanghai \\ 200011, China \\ Full list of author information is \\ available at the end of the article
}

\begin{abstract}
Background: Myocardial ischaemia reperfusion injury (MIRI) is a difficult problem in clinical practice, and it may involve various microRNAs. This study investigated the role that endogenous microRNA-146a plays in myocardial ischaemia reperfusion and explored the possible target genes.
\end{abstract}

Methods: MIRI models were established in microRNA-146a deficient (KO) and wild type (WT) mice. MicroRNA-146a expression was evaluated in the myocardium of WT mice after reperfusion. The heart function, area of myocardium infarction and in situ apoptosis were compared between the KO and WT mice. Microarray was used to explore possible target genes of microRNA-146a, while qRT-PCR and dual luciferase reporter assays were used for verification. Western blotting was performed to detect the expression levels of the target gene and related signalling molecules. A rescue study was used for further testing.

Results: MicroRNA-146a was upregulated $1 \mathrm{~h}$ after reperfusion. MicroRNA-146a deficiency decreased heart function and increased myocardial infarction and apoptosis. Microarray detected 19 apoptosis genes upregulated in the KO mice compared with the WT mice. QRT-PCR and dual luciferase verified that Med 1 was one target gene of microRNA-146a. TRAP220, encoded by Med1 in the KO mice, was upregulated, accompanied by an amplified ratio of $\mathrm{Bax} / \mathrm{BCl} 2$ and increased cleaved caspase- 3 . Inhibition of microRNA-146a in H9C2 cells caused increased TRAP220 expression and more apoptosis under the stimulus of hypoxia and re-oxygenation, while knockdown of the increased TRAP220 expression led to decreased cell apoptosis.

Conclusions: MicroRNA-146a exerts a protective effect against MIRI, which might be partially mediated by the target gene Med 1 and related to the apoptosis signalling pathway.

Keywords: Myocardial ischaemia reperfusion injury, Apoptosis, microRNA-146a, Med1

\section{Background}

Myocardial ischaemia reperfusion injury (MIRI) is a double-edged sword for myocardial infarction patients $[1,2]$. With the opening of blocked blood vessels, endangered myocardium can be saved; however, the reperfusion causes numerous free radicals and calcium overload, leading to a certain degree of myocardial injury. MIRI, which is the damage caused by reperfusion [1, 2], is undoubtedly an obstacle for the application of

(c) The Author(s). 2019 Open Access This article is distributed under the terms of the Creative Commons Attribution 4.0 International License (http://creativecommons.org/licenses/by/4.0/), which permits unrestricted use, distribution, and reproduction in any medium, provided you give appropriate credit to the original author(s) and the source, provide a link to the Creative Commons license, and indicate if changes were made. The Creative Commons Public Domain Dedication waiver (http://creativecommons.org/ publicdomain/zero/1.0/) applies to the data made available in this article, unless otherwise stated. 
reperfusion in clinical practice. Data have shown that 5-6\% of patients have cardiovascular events within 30 days after myocardial ischaemia reperfusion (MIR), which not only causes great harm to patients but also creates a heavy social burden [1]. Thus, indepth study of the occurrence, development and effective intervention measures of MIRI has become an urgent need to solve the problem.

The mechanisms of MIRI are complex and may involve various microRNAs playing protective roles, harmful roles, or double-sided roles in different stages [3-8]. For example, in vivo study has shown that inhibition of increased microRNA-24 in the infarction area can alleviate MIRI by preventing the apoptosis of cardiomyocytes $[5,6]$, whereas injection of exogenous MicroRNA-24 analogues inhibited cardiomyocyte apoptosis, thereby reducing myocardial infarct size and cardiac dysfunction $[5,7,8]$. MicroRNA-21 and microRNA-29 also play dual roles in MIRI $[4,5,9,10]$.

In our previous study, we detected the expression of microRNAs in the plasma of myocardial infarction patients who experienced percutaneous coronary intervention, and we found that microRNA-146a was one of the microRNAs that increased after reperfusion. Thus, we were interested in the role that microRNA-146a plays in MIRI. One study has reported that at 7 days before ischaemia, the injection of exogenous microRNA-146a analogues into mice myocardium was able to decrease inflammation in MIRI by targeting the TLR4-IRAK1-TRAF6 pathway [11]. However, no data have shown the expression of microRNA-146a in myocardium after MIR within 0-24 h and what will happen if endogenic microRNA-146a is lost. Furthermore, whether microRNA-146a can influence MIRI by targeting other genes or other pathways is still unclear. Therefore, this study aimed to clarify the expression and role of endogenic microRNA-146a and other mechanisms of microRNA-146a in MIRI, which will provide new insight into the treatment of MIRI.

\section{Material and methods}

Animal care

Global microRNA-146a deficient (microRNA-146a-/-, KO) mice were obtained from Jackson Laboratory, while wild-type (WT) C57BL/6 mice genetic background control mice were obtained from Shanghai SLAC Experimental Animal Co., Ltd. They were maintained in the division of Laboratory Animal Resources under specific pathogen free conditions. After the mice were bred, homozygous genotypes were identified and screened. Mice were euthanized with isoflurane over-anaesthesia. All animal experiments were approved by the Shanghai Ninth People's Hospital institutional ethics committee (HKDL2017300) and were performed in accordance with the ethical standards outlined in the 1964 Declaration of Helsinki and its later amendments.

\section{In vivo model of MIRI in mice}

Two-month-old male mice were randomly selected to be anaesthetized by $5 \%$ isoflurane inhalation, intubated and then connected to a rodent ventilator (model 683, Harvard Apparatus, Inc., USA) with $65 \%$ oxygen and anaesthesia with 3-5\% isoflurane to maintain smooth breathing in the mice, without resistance and pain responses. After the chest was opened, MIR was induced by ligating the left anterior descending (LAD) artery on the exposed heart with 7-0 silk ligature for $30 \mathrm{~min}$, followed by pulling on 
the exteriorized suture to release the knot for reperfusion. Regional ischaemia was confirmed by ECG changes (ST elevation). After reperfusion for appropriate time, hearts were harvested for qRT-PCR, Evans Blue/TTC staining, TUNEL, Gene Chip and Western blotting. Echocardiography was performed on the first and third day after reperfusion. WT and KO mice that had not experienced ligation and loosening were reviewed as sham controls.

\section{Quantitative real-time PCR (qRT-PCR) of microRNA-146a and target genes}

Total RNA (including microRNA) extraction of $\mathrm{H} 9 \mathrm{C} 2$ cells transfected with microRNA-146a inhibitor (miR20000852, RN:R10034.5, Ribobio, China) and myocardium at risk, including ischaemia and infarction tissues, using TRIZOL reagent (Qiagen) was performed in accordance with the manufacturer's protocol, followed by reverse transcription with the Thermo Cycler machine (Applied Biosystems). qRT-PCR was conducted using a real-time PCR machine (LightCycler 480 II, Roche). The microRNA-146a level was quantified by qRT-PCR using a specific assays for microRNA (Applied Biosystems, USA) and TaqMan Universal Master Mix (Applied Biosystems, USA). Specific primers for microRNA-146a were obtained from Applied Biosystems [primer identification numbers: 000468 for microRNA-146a and 001973 for U6 small nuclear RNA (snRU6)]. mRNA of the possible target genes was quantified using Takara reverse transcription assay (R0037A) and SYGRII (RR820A). Specific primers were synthesized from Sangon Biotech (Shanghai, China). The mRNA levels were quantified with the $2^{-\Delta \Delta \mathrm{cp}}$ method.

\section{Echocardiography}

Echocardiography was performed using a Vevo 770 high-resolution imaging system on the first and third day after myocardial ischaemia reperfusion. Two-dimensional and M-mode echocardiogram images were obtained after the animals were anaesthetized with isoflurane. Ejection fraction (EF) and fractional shortening (FS) were measured to evaluate the heart function.

\section{Assessment of area at risk and infarct size}

After reperfusion for $4 \mathrm{~h}$, the chest was opened again to expose the heart. The LAD was re-ligated at the previous site of ligation for staining with 1\% Evans Blue from the abdominal aorta until the whole heart turned blue. Then, the left ventricle was harvested and washed with saline. Each left ventricle was then sliced horizontally (i.e., five slices). All slices were incubated in $1 \%$ TTC for $15 \mathrm{~min}$ at $37^{\circ} \mathrm{C}$ and then fixed in $4 \%$ neutral buffered formalin for $24 \mathrm{~h}$. The white infarction and red risk areas were determined using an image analyser, corrected for the weight of each slice to sum for one whole heart. The ratios of the area at risk (AAR) to the left ventricle area (LV) and infarction size (IS) to AAR were calculated and expressed as a percentage to perform statistical analysis.

\section{Terminal deoxynucleotidyl transferase dUTP nick end labelling (TUNEL) analysis of in situ apoptosis}

At the end of reperfusion for $2 \mathrm{~h}$, the hearts were fixed with $4 \%$ buffered paraformaldehyde through abdominal aorta injection. Then, the hearts were sliced horizontally, and 
embedded in paraffin to yield several $5 \mu \mathrm{m}$ thickness sections. These sections were incubated with a prepared labelling mixture supplied by the assay (Roche, USA) at $37^{\circ} \mathrm{C}$ for $1 \mathrm{~h}$. The nuclei of living and apoptotic cells were counterstained with Hoechst 33342 (Invitrogen). Then, $40 \times$ and $400 \times$ magnification images were taken using a microscope (Nikon). The number of apoptotic cardiomyocytes was examined with the image processing software IPP6 and presented as the percentage of total cells counted.

\section{Microarray}

The Agilent Sure Print G3 Mouse GE V2.0 Microarray (8*60 K, Design ID,074809) was used. Total RNA was quantified, and the RNA integrity was assessed. The sample labelling, microarray hybridization and washing were performed based on the manufacturer's standard protocols. After washing, the arrays were scanned using the Agilent Scanner G2505C (Agilent Technologies). Feature Extraction software (version 10.7.1.1, Agilent Technologies) was used to analyse array images to obtain raw data. Genespring (version 13.1, Agilent Technologies) was used to complete the analysis with the raw data. The threshold set for the up- and down-regulated genes was a fold change $\geq 2.0$ and a $P$ value $\leq 0.05$. The microarray experiments were performed at Shanghai OE Biotech. Co., Ltd. (Shanghai, China).

\section{Dual luciferase reporter assay}

Two hundred and ninety three T cells were cultured in 24-well plates and transfected with PGL3 luciferase reporter plasmids containing wild-type or mutated mediator complex subunit 1 (Med1) 3'UTR and microRNA-146a (Genechem) using Lipo3000 reagent (Invitrogen). Cells were harvested $24 \mathrm{~h}$ later for luciferase activity detection using the DualLuciferase Reporter Assay System (Promega), according to the manufacturer's protocol.

\section{Western blotting}

After $2 \mathrm{~h}$ of reperfusion, hearts were harvested. Total protein extracted from ischaemic heart tissues with RIPA was separated by SDS-polyacrylamide gel electrophoresis and transferred onto PVDF membranes (Millipore). After being blocked with milk, the membranes were incubated with the primary antibodies anti-TRAP220 (Bethyl), antiBcl2 (CST), anti-Bax (CST), and anti-cleaved caspase-3 (CST) overnight, followed by incubation with peroxidase conjugated secondary antibodies. Analysis was conducted using the ECL system (Fusion FX7).

\section{Construction of Lenti-Med1 RNAi}

A linearized vector was obtained through digestion with restriction enzymes. Primers were annealed to prepare the desired fragment, and enzyme sites were added to the ends. Then, the vector was connected to the desired fragment that contained the same restriction sites with one another at the ends. Competent cells were transfected with the product obtained, and the monoclonal ones were selected for identification, sequencing and analysis. The correct one was expanded and extracted to obtain highpurity plasmids for virus packaging. $293 \mathrm{~T}$ cells were transfected with plasmids to obtain the target virus. After the enrichment, purification and quality inspection of virus, the construction of Lenti-Med1-RNAi was completed. 


\section{Rescue study}

H9C2 cells were cultured in 6-well plates and transfected with microRNA-146a inhibitor using lipo3000 for $48 \mathrm{~h}$ to inhibit the expression of microRNA-146a and increase the expression of TRAP220, which was encoded by the Med1 gene. In addition, the cells were infected with Lenti-Med1 RNAi for $48 \mathrm{~h}$ to decrease the expression of TRAP220. qRTPCR and Western blotting were applied to verify the effect. With the treatment above, $\mathrm{H} 9 \mathrm{C} 2$ experienced hypoxia and re-oxygenation in a hypoxia culture chamber. After that, the apoptosis of $\mathrm{H} 9 \mathrm{C} 2$ was detected with flow cytometry using Annexin V, FITC Apoptosis Detection Kit (Dojindo) according to the manufacturer's protocol.

\section{Statistical analysis}

Quantitative data were presented as the mean \pm standard deviation. Statistical significance was determined via the independent sample $t$ test between groups or ANOVA in multiple groups with SPSS 21.0 software. $P<0.050$ was considered statistically significant.

\section{Results}

MicroRNA-146a was up-regulated at an early stage of MIR

To demonstrate the expression of microRNA-146a in MIRI, WT mice were applied to build MIRI models in vivo. At different times of reperfusion, the expression of microRNA-146a was detected with qRT-PCR. We found that in the first hour after reperfusion, the expression of microRNA-146a was increased, but it declined slowly to the previous level in the next $23 \mathrm{~h}(P=0.046)$, Fig. 1 .

\section{MicroRNA-146a deficiency increased MIRI}

\section{MicroRNA-146a deficiency reduced cardiac function in MIRI}

To explicate the function of endogenous microRNA-146a in MIRI, we built the MIRI model in vivo with KO mice and WT mice, and examined cardiac function using echocardiography at the first and third day after reperfusion. As shown in Fig. 2a, no distinction of EF $(P=0.149)$ and FS $(P=0.546)$ on the first day was found. However, the

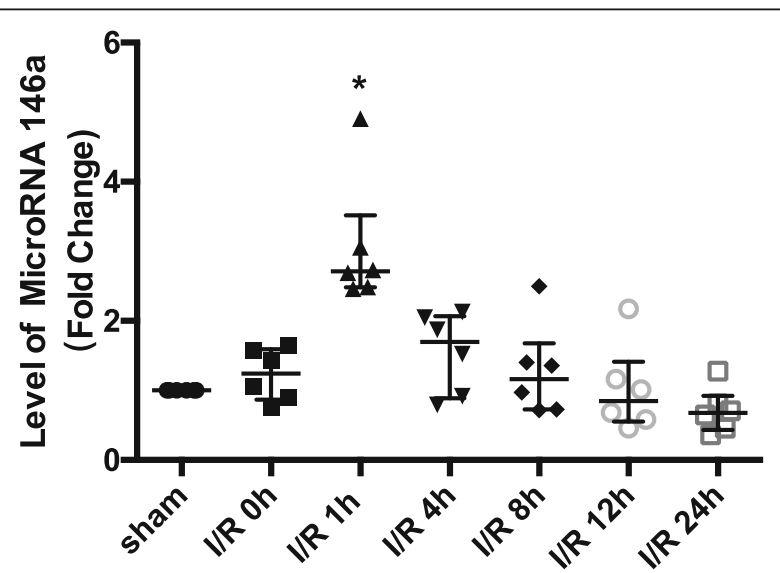

Fig. 1 MicroRNA-146a was upregulated at an early stage of myocardial ischaemia reperfusion injury (MIRI) in vivo. MicroRNA-146a expression was detected through qRT-PCR at different times after myocardial ischaemia reperfusion (MIR). Compared with the sham control group, ${ }^{*} P<0.05, n=6$ 


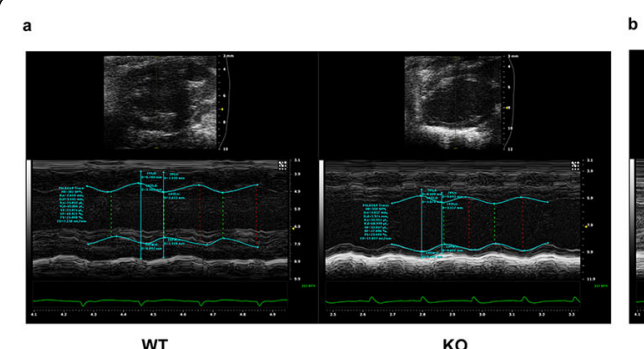

WT
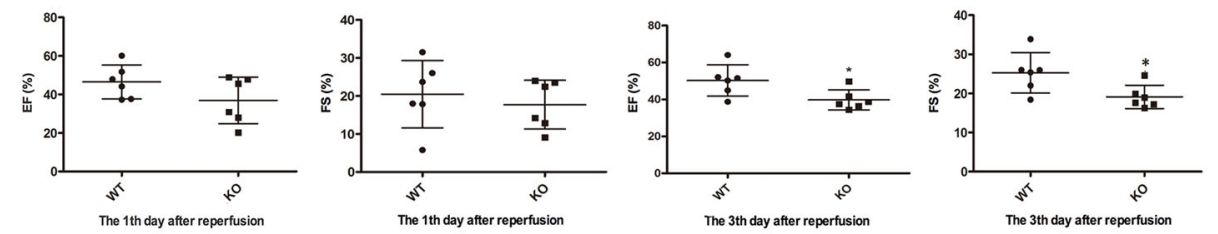

c

WT

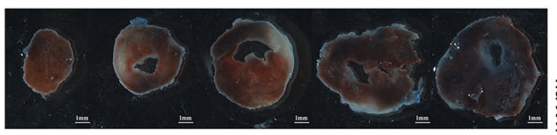

ко
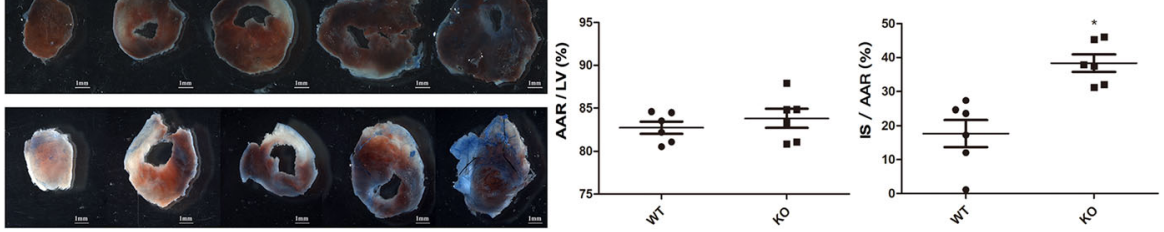

Fig. 2 MicroRNA-146a deficiency increased MIRI, a, b. MicroRNA-146a deficiency reduced cardiac function. Echocardiography was used to examine the heart function of WT and KO mice after MIR at the first and third days, EF and FS were detected. Compared with WT, ${ }^{*} P<0.05, n=6$. c MicroRNA-146a deficiency increased the myocardial infarct size. WT and KO mice were subjected to I/R, and then AAR and infarct size were subsequently measured using Evans Blue/TTC staining (10x magnification). WT, wild type; KO, microRNA-146a-/-; IR, ischaemia reperfusion; EF, ejection fraction; FS, fractional shortening; TTC, triphenyltetrazolium chloride; AAR, area at risk; IS, infarction size. Compared with WT, ${ }^{*} P<0.05, n=6$

deficiency of microRNA-146a led to the decline of EF $(P=0.028)$ and FS $(P=0.030)$ on the third day after reperfusion $(P<0.05)$, Fig. 2 b.

\section{MicroRNA-146a deficiency increased myocardial infarct size in MIRI}

We also checked the infarct and risk size of myocardium after ischaemia reperfusion in KO mice compared with WT mice. Figure $2 c$ showed that the AAR/LV in KO mice and WT mice were similar $(P=0.426)$, which demonstrated that the models were successful, and the two groups were comparable. However, IS/AAR in KO mice was much higher than that in WT mice $(P=0.002)$, meaning that microRNA-146a deficiency led to more injury in myocardial ischaemia reperfusion.

\section{MicroRNA-146a deficiency increased apoptosis in MIRI}

Apoptosis was the main cause of myocardial injury, so we measured in situ apoptosis of cardiomyocytes in vivo with TUNEL. Figure $3 \mathrm{a}$ and Fig. 3b showed that the number of apoptosis cells in the KO group was far higher than that in the WT group after MIR $(P=0.001)$. 


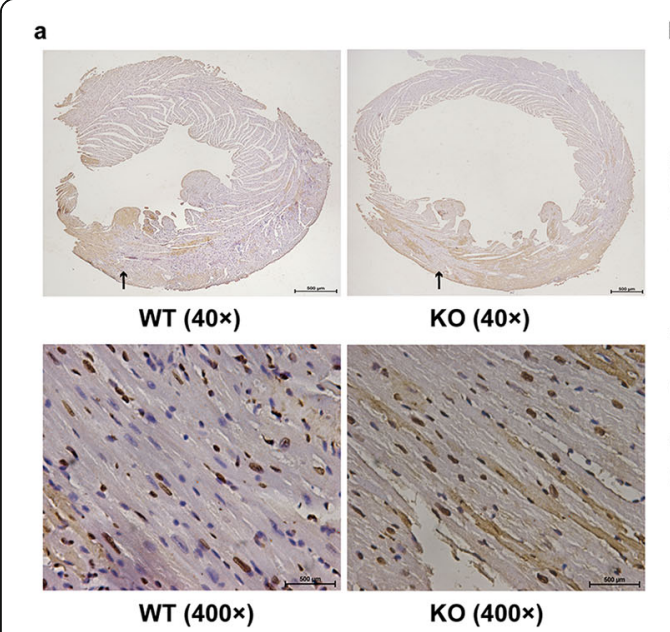

b

Fig. 3 MicroRNA-146a deficiency upregulated cardiomyocyte apoptosis in vivo after MIR. a WT and KO mice were subjected to I/R, and in situ apoptosis was determined using a TUNEL assay (Panorama above: 40× magnification; zoom below: 400x magnification). $\mathbf{b}$ An independent sample $\mathrm{t}$ test was performed for the comparison of the two groups. Compared with WT, ${ }^{*} P<0.05, n=6$

\section{Possible target genes of microRNA-146a in MIRI}

19 apoptosis related genes were detected to be the possible target of microRNA-146a

To explore new target genes of microRNA-146a, microarray was applied to distinct the difference of gene expression in myocardium between KO and WT mice after MIR. There were approximately 136 apoptosis related genes increased, including 19 genes predicted by TargetScan to be able to combine with microRNA-146a, which are shown in the heatmap in Fig. $4 \mathrm{a}$.

\section{MicroRNA-146a deficiency increased 5 apoptosis related genes' mRNA expression after MIRI}

With the preliminary results of microarray and TargetScan, we made further verification through qRT-PCR in myocardium of KO and WT mice that experienced myocardial ischaemia reperfusion. Figure $4 \mathrm{~b}$ shows that, in the 19 apoptosis related genes, mRNA expression of 5 genes was upregulated: IL2rb $(P=0.323)$, Lsp1 $(P=$ 0.875), Aldh2 $(P=0.295)$, Dapk3 $(P=0.009)$ and Med1 $(P=0.000)$. Med1 was the gene with the highest expression. Thus, it was the best candidate target gene for microRNA-146a in MIRI.

\section{Med1 was one target gene of microRNA-146a}

We chose Med1 with the highest mRNA expression to check if it was the target gene of microRNA-146a using dual luciferase reporter in $293 \mathrm{~T}$ cells. After transfection with PGL3 luciferase reporter plasmids containing wild-type or mutated Med1 3'UTR and microRNA-146a, as shown in Fig. 4c, the fluorescence intensity in the microRNA-146a + Med1 WT-3'UTR group was significantly lower than that in other groups $(P=0.000)$, demonstrating that Med1 was the target gene of microRNA-146a. 


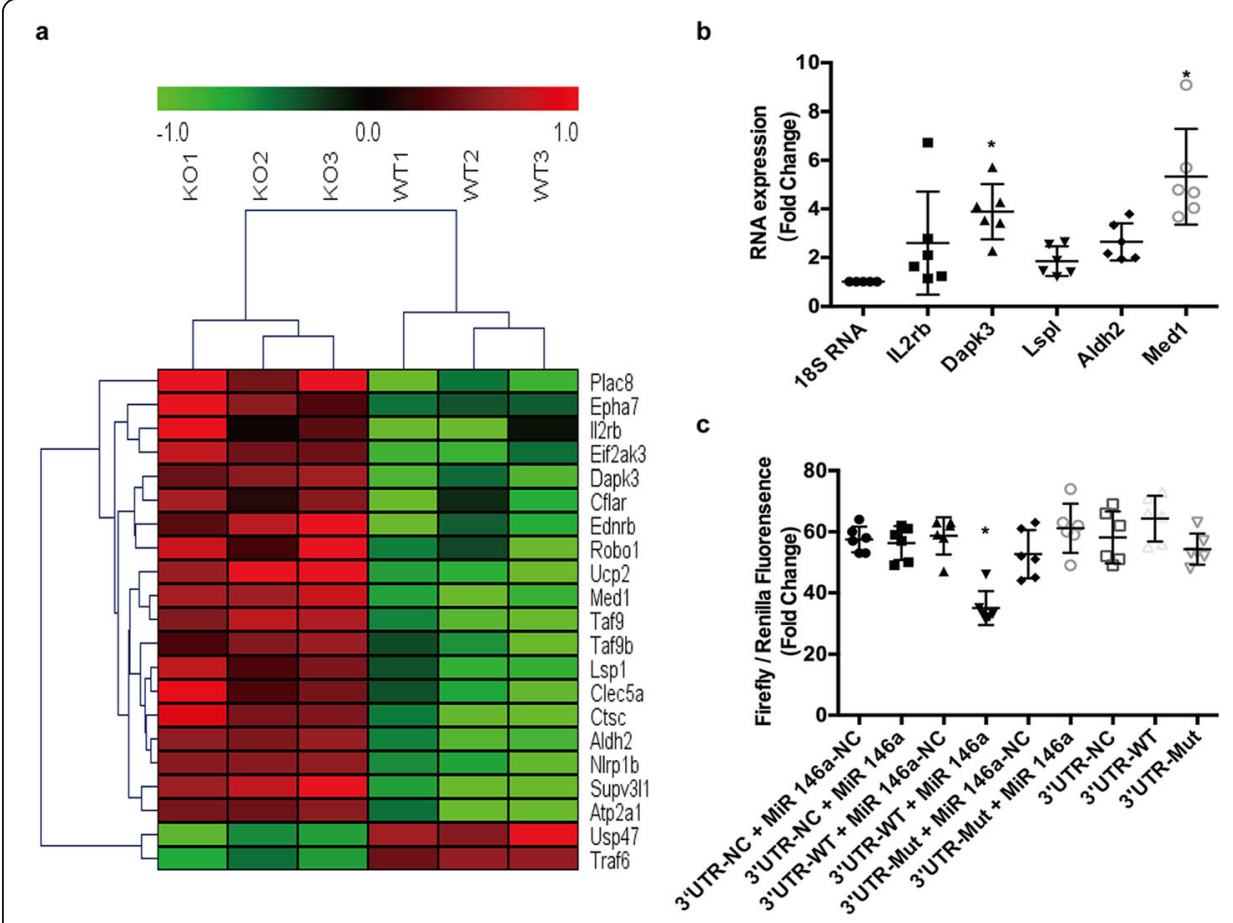

Fig. 4 Med 1 was one of the target genes of microRNA-146a. a WT and KO mice were subjected to MIR, and then possible target genes were checked with microarray. The heatmap showed 19 apoptosis related genes were up-regulated. $\mathbf{b}$ WT and KO mice were stimulated with MIR, and the mRNA levels of IL2rb, Dapk3, Lspl, Aldh2, and Med1 were detected through gRT-PCR, with $18 \mathrm{~s}$ RNA as an internal reference. Compared with $18 \mathrm{~s}$ RNA, ${ }^{*} P<0.05, n=6$. c 293 T cells were transfected with $P G L 3$ luciferase reporter plasmids containing wild-type or mutated Med1 $3^{\prime} U T R$ and microRNA-146a, using NC as controls. NC, negative control. Compared with other groups, ${ }^{*} P<0.05, n=6$

\section{MicroRNA-146a deficiency increased TRAP220, accompanied by aggravated apoptosis} protein expression in myocardium

To explore the possible mechanism of microRNA-146a and Med1 in MIRI, apoptosis related protein was detected through Western blotting analysis. As shown in Fig. 5a and $b$, we found that TRAP220 was increased when myocardium experienced MIR, compared with the sham control group $(P=0.002)$. Under the same condition of ischaemia reperfusion, myocardial microRNA-146a deficiency led to a greater increase of TRAP220, which was encoded by Med1, compared to WT. At the same time, apoptosis related protein Bax and cleaved caspase-3 were also upregulated $(P=0.000)$. However, the expression of Bcl2 was decreased $(P=0.004)$, which suggested that the lack of microRNA-146a could cause increased expression of TRAP220, accompanied by an amplified ratio of $\mathrm{Bax} / \mathrm{Bcl} 2$ and increased cleaved caspase-3.

MicroRNA-146a decreased the apoptosis of $\mathrm{H} 9 \mathrm{C} 2$ with stimulation of hypoxia and reoxygenation by targeting Med1

To further confirm whether microRNA-146a can influence apoptosis in MIRI partly through targeting Med1, a rescue study of $\mathrm{H} 9 \mathrm{C} 2$ cells was conducted. As shown in Fig. 6a and b, we found that microRNA-146a inhibitor decreased the expression of microRNA-146a $(P=0.000)$ while it increased TRAP220 protein $(P=0.000)$ in H9C2. 


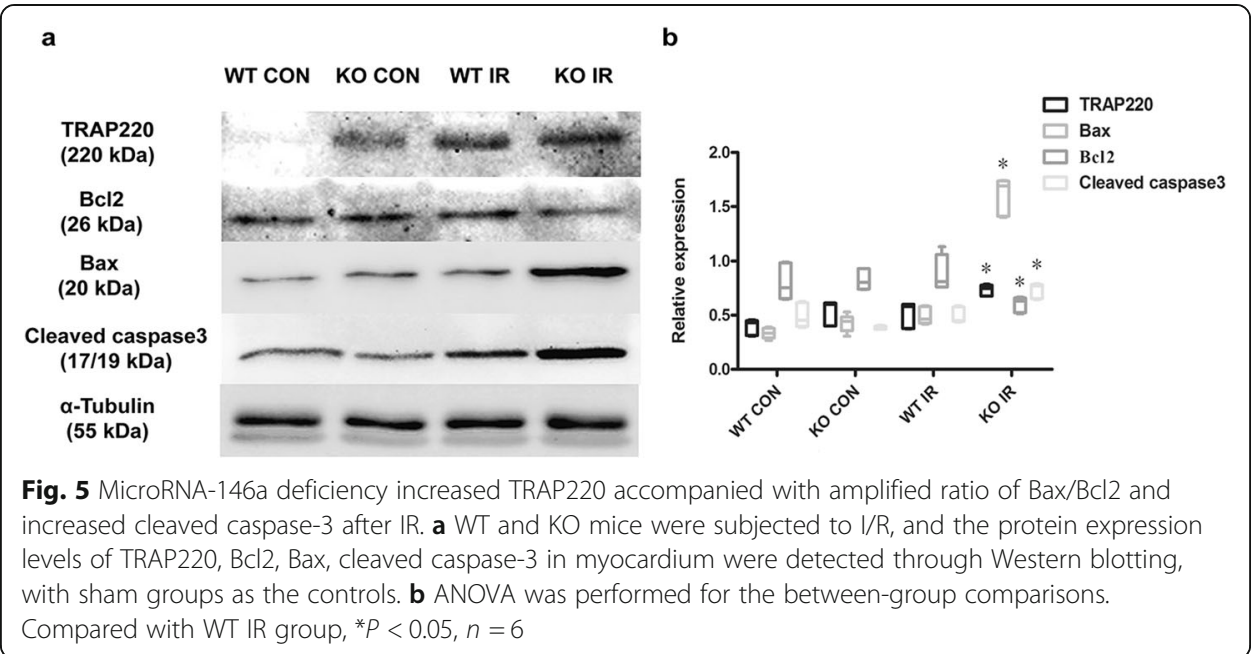

Lenti-Med1-RNAi successfully suppressed the upregulation of TRAP220 $(P=0.000)$ (Fig. 6c). The flow cytometry results (Fig. 6d) demonstrated that the apoptosis of $\mathrm{H} 9 \mathrm{C} 2$, which experienced hypoxia and re-oxygenation, was greater than that of the control group $(P=0.000)$. When the expression of microRNA-146a was suppressed, the apoptosis ratio was further increased $(P=0.018)$. However, with the downregulation of Med1, after the inhibition of microRNA-146a in H9C2 cells that were stimulated with hypoxia and re-oxygenation, the apoptosis ratio decreased greatly $(P=0.000)$. This result suggested that microRNA-146a did decrease the apoptosis of H9C2 stimulated with hypoxia and re-oxygenation, partly by targeting Med1.

\section{Discussion}

In the present study, we examined the expression and role of endogenous microRNA146a in MIRI, and we explored the possible target genes and pathways. Our results indicated that microRNA-146a was upregulated at an early stage of MIR, and endogenic microRNA-146a deficiency increased MIRI, demonstrating a protective role in this process. The role of endogenic microRNA-146a was consistent with that of exogenous microRNA-146a mimics previously reported, which also proved that the increase of microRNA-146a in the first hour after reperfusion was a compensatory protection initiated by the body itself. However, this compensatory protective effect slowly disappeared after that. Therefore, upregulation of microRNA-146a occurs as early as the reperfusion occurs and as long as the injury duration would be beneficial for ischaemic myocardium. Thus, interventions that can increase microRNA-146a, such as drugs, surgery or direct exogenous addition of microRNA-146a mimics at a suitable time, can be used to protect ischaemic myocardium after reperfusion.

MicroRNA is a class of non-coding single-stranded RNA molecules encoded by endogenous genes of approximately 22 nucleotides [3, 12, 13]. They are capable of completely or incompletely binding to the target genes and then degrading them or modulating their functions $[3,12,13]$. It has been demonstrated that microRNA-146a can target many genes, such as IRAK1, IRAK2, TRAF6, RIG-I, IRF-5, STAT-1, PTC1, Numb, and WASF2, to play a variety of roles in human diseases, including cancers and inflammatory immune diseases [14-18]. IRAK1 and TRAF6 are the downstream 


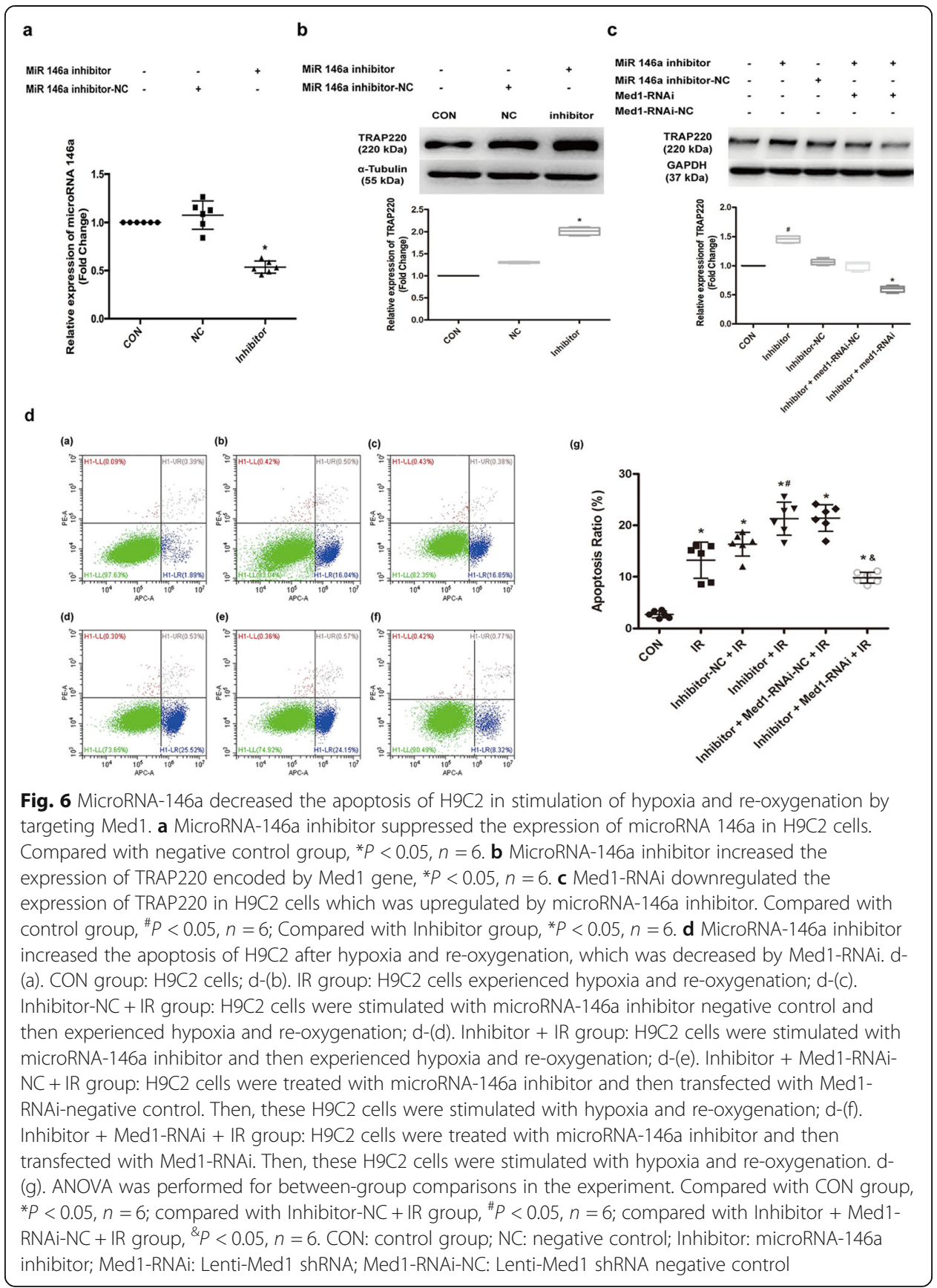

molecules of the TLR-induced NF- $\mathrm{KB}$ signalling pathway, and they have often been identified as the target genes of microRNA-146a in inflammatory immune diseases containing MIRI [11, 19-21]. However, a few other target genes of microRNA-146a in MIRI are known. In this study, we found that mediator complex subunit 1 (Med1) was one target gene of microRNA-146a, and TRAP220 protein encoded by Med1 was upregulated when microRNA-146a was deficient.

The multi-subunit mediator is an evolutionarily conserved transcription coregulatory nuclear complex in eukaryotes. It is needed for the transcription regulation of gene expression in general, as well as in a gene specific manner. Mediator complex 
subunits interact with different transcription factors and as components of the RNA Pol II transcription initiation complex; in doing so, they act as a bridge between gene specific transcription factors and general Pol II transcription machinery [22]. Med1 is also known as TRAP220 in mice and RB18A in humans. Researchers have reported that cardiac Med1 was necessary for survival in mice because it regulated cardiac metabolic and mitochondrial genes. Genetic deletion of Med1 resulted in embryonic lethality, largely due to impaired cardiac development [23, 24]. Other studies have also shown that cardiomyocyte-specific ablation of the Med1 subunit of the mediator complex might cause lethal dilated cardiomyopathy [25]. However, no data detailing whether Med1 can influence MIRI are available. In our study, we found that the upregulation of TRAP220 in microRNA-146a deficient mice that experienced myocardial ischaemia reperfusion was accompanied by an amplified ratio of $\mathrm{Bax} / \mathrm{Bcl} 2$ and increased cleaved caspase-3. The rescue study verified that Med1 was indeed one target gene of microRNA-146a in MIRI.

Apoptosis is a sophisticated process that is the main cause of MIRI [26]. There are two main pathways involved in apoptosis: the extrinsic (death receptor) pathway and the intrinsic (mitochondrial) pathway [27, 28]. However, the last stage of both pathways is initiated by the cleavage of caspase- 3 and results in DNA fragmentation, degradation of cytoskeletal and nuclear proteins, cross-linking of protein, formation of apoptotic bodies, expression of ligands for phagocytic cell receptors and finally, uptake by phagocytic cells. In the intrinsic pathway, stimuli, including radiation, toxins, viral infections, hyperthermia, hypoxia and free radicals, are able to cause changes in the inner mitochondrial membrane, which may result in an opening of the mitochondrial permeability transition pore and loss of the mitochondrial transmembrane potential. Then, two main groups of normally sequestered pro-apoptotic proteins are released from the intermembrane space into the cytosol, consisting of cytochrome c, Smac/DIABLO, and the serine protease HtrA2/Omi, which will activate the caspase-dependent mitochondrial pathway. Cytochrome c initiates activation of a series of kinases, including caspase-3, which is the ultimate executor of apoptosis [27, 28]. These apoptotic mitochondrial events can be regulated by the Bcl-2 family proteins, such as anti-apoptotic proteins Bcl- 2 and pro-apoptotic proteins Bax, which control the release of cytochrome c from the mitochondria via alteration of mitochondrial membrane permeability [27, 28]. p53, a tumour suppressor protein, has a critical role in regulation of the Bcl-2 family [27-31]. The p53 tumour suppressor gene is a transcription factor that regulates the cell cycle. It can activate DNA repair proteins when DNA has sustained damage, hold the cell cycle at the G1/S regulation point on DNA damage recognition, and initiate apoptosis if the DNA damage proves to be irreparable. p53 is one regulator of Bcl2 and Bax [2731]. Researchers have revealed that RB18A, a member of the Med1 family, selfoligomerized and interacted with the p53 protein in vitro when K562 (an erythroleukaemia cell line) and H1299 (pulmonary embryo carcinoma) cells, two human p53-null cell lines, were used to transfect with p53wt cDNA in the presence or absence of RB18A cDNA [29, 30]. In addition, with immunoprecipitation, they demonstrated that in vivo, RB18A directly interacted (through its C-terminal domain) with wild type p53 (p53 wt), acting as a cofactor of transcription by directly regulating p53-transactivating activity on the promoters of Bax, p21Waf1 and IGF-BP3 genes [29, 30], while RB18A alone did not affect cell apoptosis. In this process, RB18A activated Bax promoter and 
inhibited p21Waf1 and IGF-BP3 promoters [29, 30, 32]. TRAP220, a 220 KDa thyroid hormone receptor-associated protein, which shared $99 \%$ sequence identity within the RB18A coding sequence (with only minor sequence variations), was also demonstrated to interact with p53 wt as RB18A [29, 30, 33]. In our study, we inferred that the increased TRAP220 protein in the $\mathrm{KO}$ mice that experienced myocardial ischaemia reperfusion might interact with p53 protein, activate the promoter of Bax, and then lead to the cleavage of caspase 3 , which mediated apoptosis.

Our study is the first to clarify the expression and role of endogenous microRNA146a after MIRI. We also found one new target gene of microRNA-146a, Med1, which might mediate apoptosis through regulating the p53 related signal pathway in MIRI. However, more studies are still needed to verify the apoptosis signal pathway in this process.

\section{Conclusion}

In conclusion, microRNA-146a may demonstrate compensatory upregulation in the early stage of MIR, and it possesses a protective effect against MIRI, which might be partially mediated by the target gene Med1 and related apoptosis signal pathway. Thus, it is suggested to increase the expression of microRNA-146a at the initial stage of myocardial ischaemia reperfusion as early as possible through interventions, such as drugs, surgery or direct exogenous addition of microRNA-146a mimics, to better protect the heart.

\section{Abbreviations}

AAR: Ratio of the area at risk; EF: Ejection fraction; FS: Fractional shortening; IS: Infarction size; KO: microRNA-146a deficient; LAD: Left anterior descending artery; LV: Left ventricle area; Med1: Mutated mediator complex subunit 1; MIR: Myocardial ischaemia reperfusion; MIRI: Myocardial ischaemia reperfusion injury; qRT-PCR: quantitative real-time PCR; WT: Wild type

\section{Acknowledgements}

We thank Shanghai OE Biotech. Co., Ltd. (Shanghai, China) for the microarray experiment.

\section{Authors' contributions}

CW and JZ designed and guided this study. TZ and YM conducted all the experiments. LG, CM, HZ, XW and YS participated in cell experiments. JG, YW, KC, ZH, YF and JG participated in the animal experiments. All authors read and approved the final manuscript.

Funding

This work was supported by Chinese National Natural Science Foundation Grant (Grant No. 81870264, 81670316), the Shanghai Shenkang Hospital Development Center of China Grant (Grant No. 16CR2034B) and Shanghai Ninth People's Hospital affiliated to Shanghai Jiao Tong University Grant (Grant No. JYLJ017).

\section{Availability of data and materials}

The datasets used and/or analysed during the current study are available from the corresponding author on reasonable request.

\section{Ethics approval and consent to participate}

All animal experiments were approved by Shanghai Ninth People's Hospital institutional ethics committee (HKDL2017300) and were performed in accordance with the ethical standards laid down in the 1964 Declaration of Helsinki and its later amendments.

Consent for publication

Not applicable.

Competing interests

The authors declare that they have no competing interests.

\section{Author details}

'Department of Cardiology, Shanghai Ninth People's Hospital, Shanghai Jiao Tong University School of Medicine,

Shanghai 200011, China. 'Department of Anaesthesiology, Shanghai Ninth People's Hospital, Shanghai Jiao Tong 
University School of Medicine, Shanghai 200011, China. ${ }^{3}$ Department of Cardiovascular Surgery, Renji Hospital, School of Medicine, Shanghai Jiao Tong University, Shanghai 200127, China.

Received: 5 July 2019 Accepted: 5 November 2019

Published online: 27 November 2019

\section{References}

1. Hausenloy DJ, Yellon DM. Ischaemic conditioning and reperfusion injury. Nat Rev Cardiol. 2016;13(4):193-209.

2. Braunwld E, Kloner RA. Myocardial reperfusion: a double-edged sword? J Clin Invest. 1985;76(5):1713-9.

3. Beermann J, Piccoli MT, Viereck J, Thum T. Non-coding RNAs in development and disease: background, mechanisms, and therapeutic approaches. Physiol Rev. 2016;96(4):1297-325.

4. Ye Y, Perez-Polo JR, Qian J, Birnbaum Y. The role of microRNA in modulating myocardial ischemia-reperfusion injury. Physiol Genomics. 2011:43(10):534-42.

5. Fan ZX, Yang J. The role of microRNAs in regulating myocardial ischemia reperfusion injury. Saudi Med J. 2015;36(7): 787-93.

6. Fiedler J, Jazbutyte V, Kirchmaier BC, Gupta SK, Lorenzen J, Hartmann D, et al. MicroRNA-24 regulates vascularity after myocardial infarction. Circulation. 2011;124(6):720-30.

7. Qian L, Van Laake LW, Huang Y, Liu S, Wendland MF, Srivastava D. miR-24 inhibits apoptosis and represses Bim in mouse cardiomyocytes. J Exp Med. 2011;208(3):549-60.

8. Guo C, Deng Y, Liu J, Qian L. Cardiomyocyte-specific role of miR-24 in promoting cell survival. J Cell Mol Med. 2015; 19(1):103-12.

9. Xu X, Kriegel AJ, Jiao X, Liu H, Bai X, Olson J, et al. miR-21 in ischemia/reperfusion injury: a double-edged sword? Physiol Genomics. 2014;46(21):789-97.

10. Ślusarz A, Pulakat L. The two faces of miR-29. J Cardiovasc Med (Hagerstown). 2015;16(7):480-90.

11. Wang X, Ha T, Liu L, Zou J, Zhang X, Kalbfleisch J, et al. Increased expression of microRNA-146a decreases myocardial ischaemia/reperfusion injury. Cardiovasc Res. 2013;97(3):432-42.

12. Hammond SM. An overview of microRNAs. Adv Drug Deliv Rev. 2015;87:3-14.

13. Mohr AM, Mott JL. Overview of microRNA biology. Semin Liver Dis. 2015;35(1):3-11.

14. Li L, Chen XP, Li YJ. MicroRNA-146a and human disease. Scand J Immunol. 2010;71(4):227-31.

15. Paterson MR, Kriegel AJ. MiR-146a/b: a family with shared seeds and different roots. Physiol Genomics. 2017;49(4):24352.

16. Xu WD, Lu MM, Pan HF, Ye DQ. Association of MicroRNA-146a with autoimmune diseases. Inflammation. 2012;35(4): $1525-9$.

17. Yao Q, Cao Z, Tu C, Zhao Y, Liu H, Zhang S. MicroRNA-146a acts as a metastasis suppressor in gastric cancer by targeting WASF2. Cancer Lett. 2013;335(1):219-24.

18. Zhang X, Li D, Li M, Ye M, Ding L, Cai H, et al. MicroRNA-146a targets PRKCE to modulate papillary thyroid tumor development. Int J Cancer. 2014;134(2):257-67.

19. Ramkaran P, Khan S, Phulukdaree A, Moodley D, Chuturgoon AA. miR-146a polymorphism influences levels of miR-146a, IRAK-1, and TRAF-6 in young patients with coronary artery disease. Cell Biochem Biophys. 2014;68(2):259-66.

20. Zilahi E, Tarr T, Papp G, Griger Z, Sipka S, Zeher M. Increased microRNA-146a/b, TRAF6 gene and decreased IRAK1 gene expressions in the peripheral mononuclear cells of patients with Sjögren's syndrome. Immunol Lett. 2012;141(2):165-8.

21. Lv F, Huang Y, Lv W, Yang L, Li F, Fan J, et al. MicroRNA-146a ameliorates inflammation via TRAF6/NF-KB pathway in intervertebral disc cells. Med Sci Monit. 2017;23:659-64.

22. Ranjan A, Ansari SA. Therapeutic potential of Mediator complex subunits in metabolic diseases. Biochimie 2017; S03009084(17)30264-X.

23. Spitler KM, Ponce JM, Oudit GY, Hall DD, Grueter CE. Cardiac Med1 deletion promotes early lethality, cardiac remodeling, and transcriptional reprogramming. Am J Physiol Heart Circ Physiol. 2017;312(4):H768-80.

24. Schiano C, Casamassimi A, Vietri MT, Rienzo M, Napoli C. The roles of mediator complex in cardiovascular diseases. Biochim Biophys Acta. 2014;1839(6):444-51.

25. Jia Y, Chang HC, Schipma MJ, Liu J, Shete V, Liu N, et al. Cardiomyocyte-specific ablation of Med1 subunit of the mediator complex causes lethal dilated cardiomyopathy in mice. PLoS One. 2016;11(8):e0160755.

26. Gottlieb RA. Cell death pathways in acute ischemia/reperfusion injury. J Cardiovasc Pharmacol Ther. 2011;16(3-4):233-8.

27. Elmore S. Apoptosis: a review of programmed cell death. Toxicol Pathol. 2007;35(4):495-516.

28. Nagata S, Tanaka M. Programmed cell death and the immune system. Nat Rev Immunol. 2017;17(5):333-40.

29. Frade $\mathrm{R}$, Balbo M, Barel M. RB18A, whose gene is localized on chromosome 17q12-q21.1, regulates in vivo p53 transactivating activity. Cancer Res. 2000;60(23):6585-9.

30. Frade R, Balbo M, Barel M. RB18A regulates p53-dependent apoptosis. Oncogene. 2002;21(6):861-6.

31. Yoshida K, Miki Y. The cell death machinery governed by the p53 tumor suppressor in response to DNA damage. Cancer Sci. 2010;101:831-5.

32. Karimian A, Ahmadi Y, Yousefi B. Multiple functions of p21 in cell cycle, apoptosis and transcriptional regulation after DNA damage. DNA Repair (Amst). 2016;42:63-71.

33. Ito M, Yuan CX, Malik S, Gu W, Fondell JD, Yamamura S, et al. Identity between TRAP and SMCC complexes indicates novel pathways for the function of nuclear receptors and diverse mammalian activators. Mol Cell. 1999;3(3):361-70.

\section{Publisher's Note}

Springer Nature remains neutral with regard to jurisdictional claims in published maps and institutional affiliations. 